\title{
Coupling effect on the proton optics from the electron lenses
}

\author{
Y. Luo, X. Gu, W. Fischer \\ Brookhaven National Laboratory, Upton, NY 11973, USA
}

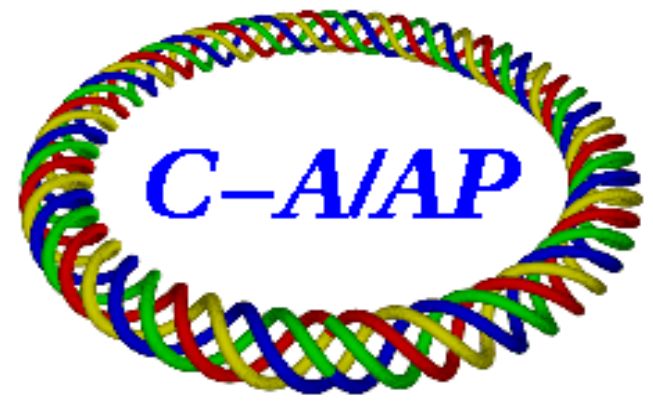

\section{Collider-Accelerator Department Brookhaven National Laboratory Upton, NY 11973}

Notice: This document has been authorized by employees of Brookhaven Science Associates, LLC under Contract No. DE-AC02-98CH10886 with the U.S. Department of Energy. The United States Government retains a nonexclusive, paid-up, irrevocable, world-wide license to publish or reproduce the published form of this document, or allow others to do so, for United States Government purposes. 
June 15, 2010

\title{
Coupling effect on the proton optics from the electron lenses
}

\author{
Y. Luo, X. Gu, W. Fischer \\ Brookhaven National Laboratory, Upton, NY 11973, USA
}

In this note we calculate the effect of the electron lense solenoids on the proton optics. Electron lenses ( e-lenses ) are to be used for head-on beam-beam compensation in the Relativistic Heavy Ion Collider (RHIC).

\section{Introduction}

Electron lenses are to be used for head-on beam-beam compensation in the polarized proton (pp) runs to compensate the large tune spread generated by the head-on proton-proton beam-beam interactions at IP6 and IP8 in the Relativistic Heavy Ion Collider (RHIC) [1]. The main part of an electron lens is a superconducting solenoid with a longitudinal magnetic field up to $6 \mathrm{~T}$.

In the current design of RHIC head-on beam-beam compensation, there are two e-lenses, one for the Blue ring called BEL and one for the Yellow ring called YEL. The effective length of these e-lens solenoids is $2.0 \mathrm{~m}$. They are located $1.5 \mathrm{~m}$ away from IP10. The actual operating solenoid field may range from $3 \mathrm{~T}$ to $6 \mathrm{~T}$. Figure 1 shows the layout of RHIC head-on beam-beam compensation at $250 \mathrm{GeV}$. Figure 2 shows the locations of e-lenses around IP10.

As we know, solenoids will introduce betetron coupling into the proton linear optics. It will couple the horizontal orbit into the vertical plane. If there is horizontal dispersion in the solenoid, it will generate dispersion in the vertical plane. Beside the local coupling effect, the solenoid also will increase the eigen tune split. To cancel the effects of the two solenoids on the betatron coupling, in the current design of RHIC head-on beam-beam compensation, the two solenoids will be powered to have opposite magnetic fields.

In the following, we will estimate the e-elenses' effects on the $\beta$ and dispersion functions with $100 \mathrm{GeV}$ and $250 \mathrm{GeV}$ pp run lattices. Table 1 lists some lattice and beam parameters to be used in the following study.

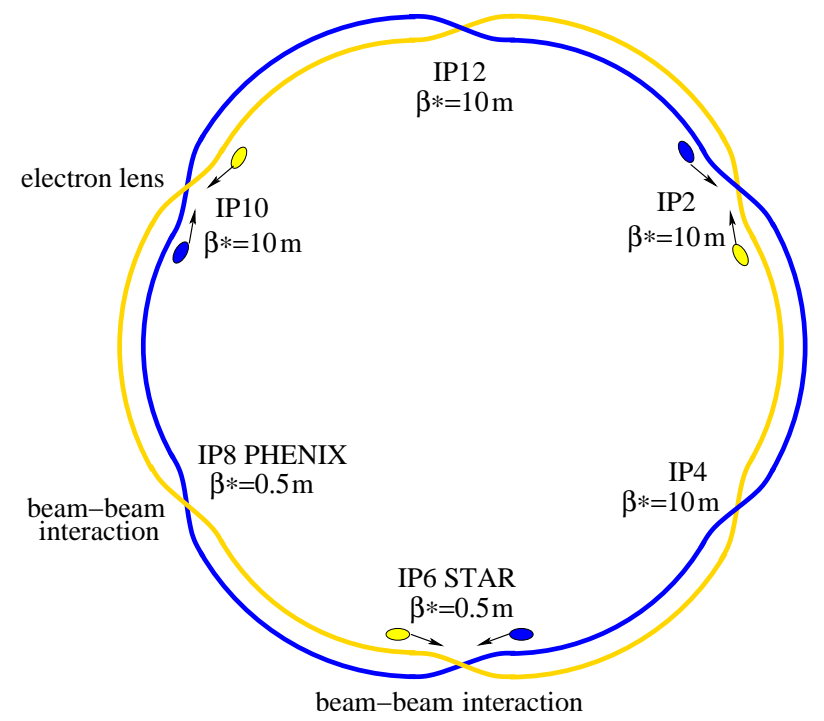

Figure 1: Layout of RHIC head-on beam-beam compensation. 


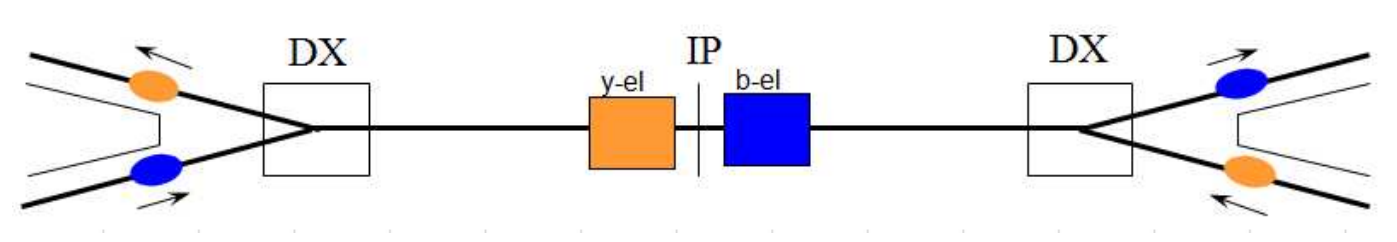

Figure 2: RHIC e-lenses around IP10.

Table 1: Lattice and beam parameters used in this study

\begin{tabular}{lcc}
\hline \hline parameter & 100 GeV lattice & 250 GeV lattice \\
\hline proton relativistic $\gamma$ & 106 & 266 \\
$\beta_{x, y}^{*}$ at IP6 and IP8 & $0.8 \mathrm{~m}$ & $0.5 \mathrm{~m}$ \\
$\beta_{x, y}^{e}$ at e-lens & $10 \mathrm{~m}$ & $10 \mathrm{~m}$ \\
normalized transverse rms emittance $\epsilon_{x, y}$ & \multicolumn{2}{c}{$2.5 \mathrm{~mm} . \mathrm{mrad}$} \\
non-colliding transverse tunes & \multicolumn{2}{c}{$(28.685,29.695)$} \\
linear chromaticities & \multicolumn{2}{c}{$(1,1)$} \\
proton transverse rms beam size at IP10 & $0.48 \mathrm{~mm} \quad 0.30 \mathrm{~mm}$ \\
\hline \hline
\end{tabular}

\section{Effects on $\beta$ function and coupling parameter $r$}

First we calculate the effects of the two solenoids on the $\beta$ function and coupling parameter $r$ along the ring. As we know, $r^{2}+\|\mathbf{C}\|=1$, where $\mathbf{C}$ is the coupling matrix. The eigen emittance exchange between the two eigen modes are proportional to $1-r^{2} / r^{2}[2,3]$.

In this calculation, we found that there is no significant changes in the eigen $\beta$ functions and the horizontal dispersion along the ring.

Figure 3 and Figure 4 shows the coupling parameter $r$ along the ring with opposite and same polarities of the two e-elens solenoids. Here $100 \mathrm{Gev}$ pp run lattice is used. With the opposite polarities of the two solenoids, the effects on the local coupling parameter is localized in the ring and is neglectible. With same polarities of the two solenoids, the coupling can be seen in the whole ring and its ampitude is about 0.97 .

In the Appendix, we show the $\beta$ functions and coupling parameter $r$ in the scan of solenoid field amplitude for the $100 \mathrm{GeV}$ and $2500 \mathrm{GeV}$ pp run lattices. The solenoids are powered with same or different polarities, and even with one solenoid off.

\section{$3 \quad$ Effect on vertical disperison}

Figure 5 and Figure 6 show the vertical dispersion along the ring due to the e-lens solenoids with $250 \mathrm{GeV}$ and $100 \mathrm{GeV}$ pp run lattices. The solenoid magnetic field is set to $6 \mathrm{~T}$. We calculate different polarity combinations of the two e-lens solenoids.

From Figure 5 and Figure 6, with opposite polarities of the two solenoids, the vertical disperson is localized between the two solenoids. With same polarities of the two solenoids, the vertical disperson leaks out to the rest of the ring.

Also from Figure 5 and Figure 6, with opposite solenoid polarities, the maximum vertical disperison is less than $1 \mathrm{~mm}$ for the $100 \mathrm{GeV}$ pp lattice. However, with same solenoid polarity, the maximum vertical disperison is about $7 \mathrm{~mm}$ and $3.5 \mathrm{~mm}$ in the trplets of IR6 and IR 8 for the $100 \mathrm{GeV}$ and $250 \mathrm{GeV}$ lattices.

\section{Effect on dQmin}

With betaron coupling, there is a minimum eigen tune split, which we call dQmin. As we know, the fractional tune split is given by [4]

$$
\left|Q_{1}-Q_{2}-p\right|=\sqrt{\left|Q_{x, 0}-Q_{y, 0}-p\right|+\mid C^{-1}},
$$

where $\left|Q_{1}-Q_{2}-p\right|$ and $\left|Q_{x, 0}-Q_{y, 0}-p\right|$ are fractional eigen tune split and set tune split, $\left|C^{-}\right|$is global coulpling coefficient. 


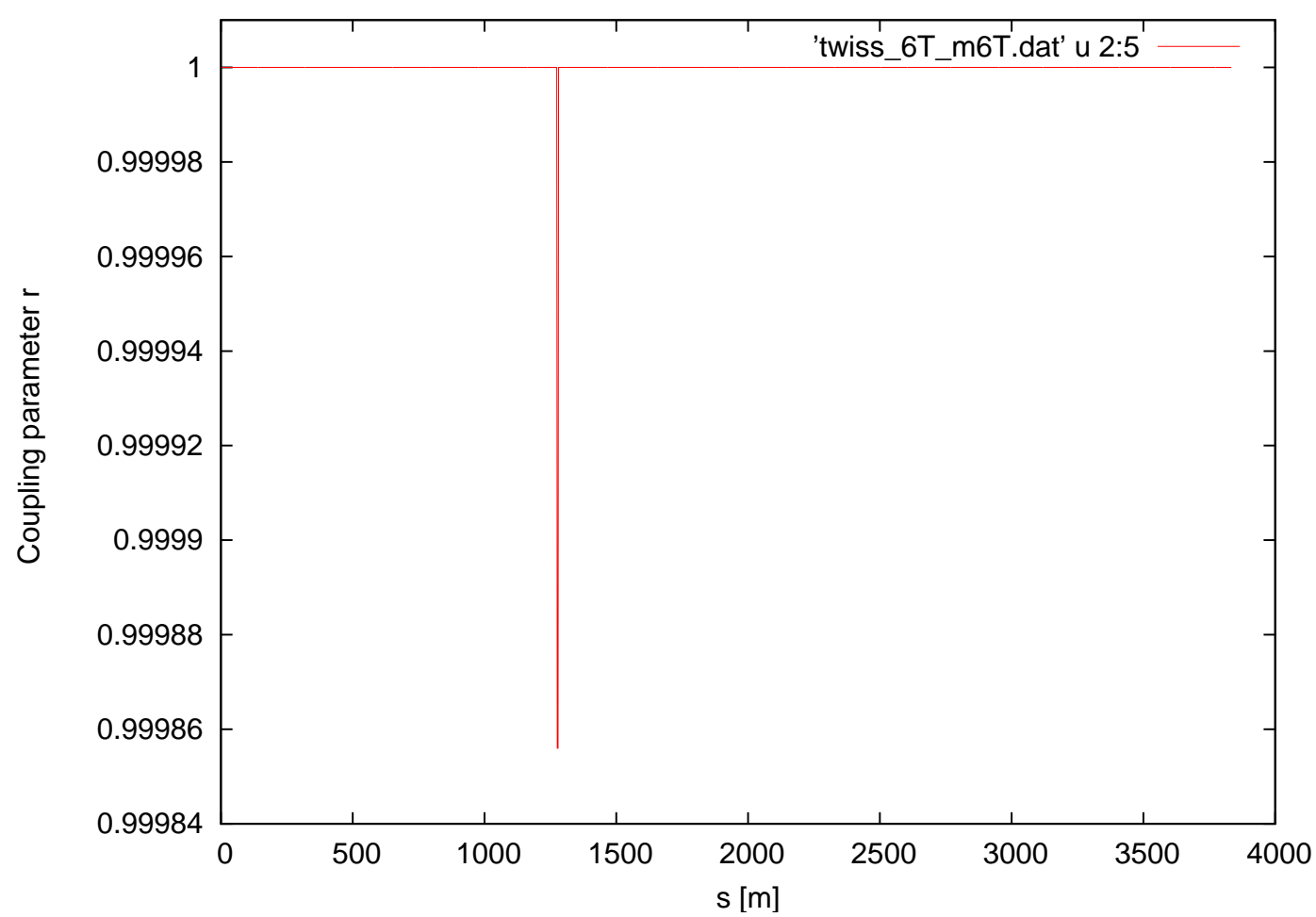

Figure 3: $100 \mathrm{GeV}$ : coupling parameter $r$ along the ring with opposite soleoind polarities.

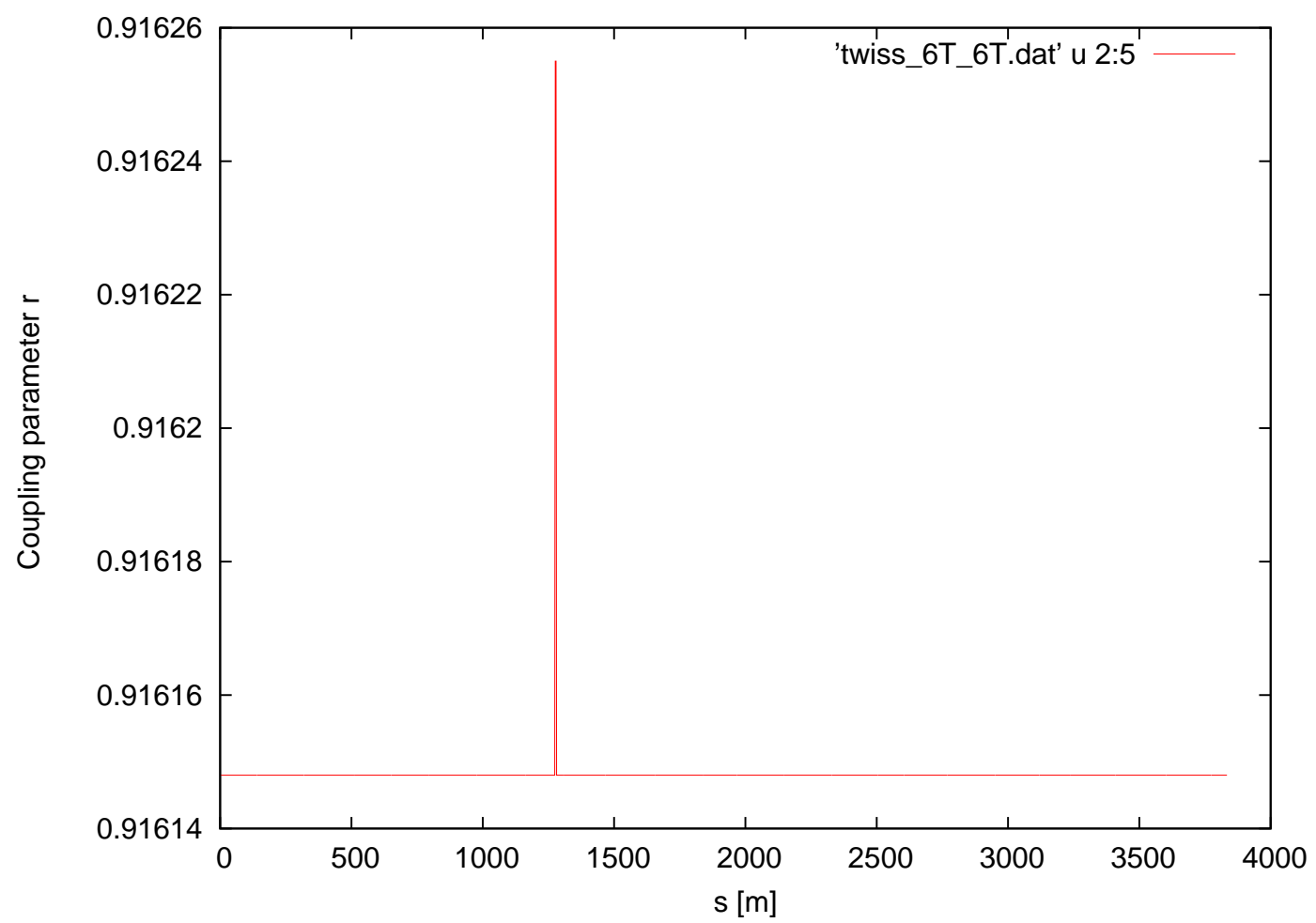

Figure 4: $100 \mathrm{GeV}$ : coupling parameter $r$ along the ring with same soleoind polarities. 


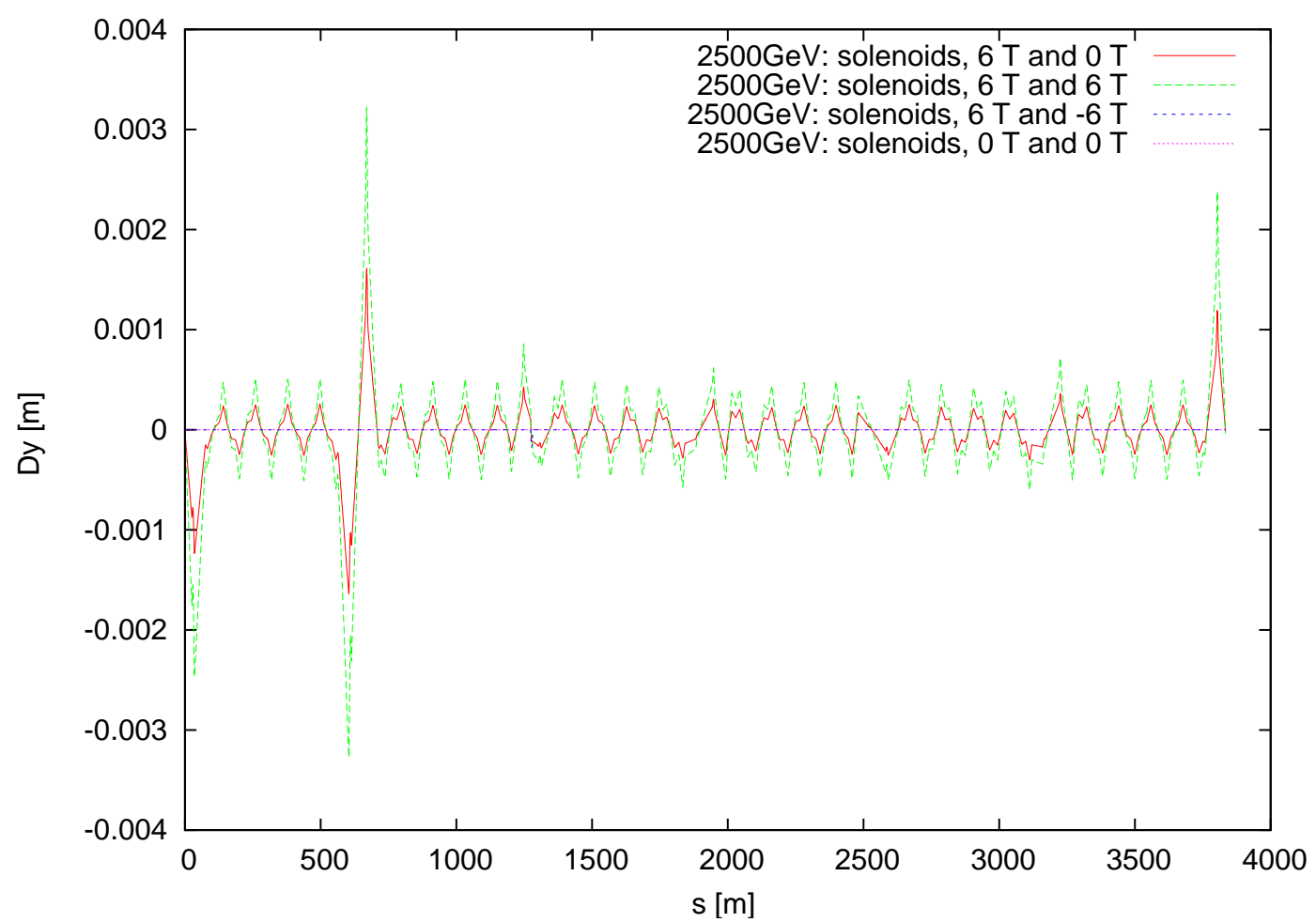

Figure 5: $250 \mathrm{GeV}$ : Vertical dispersion along the ring with different soleoind powering cases

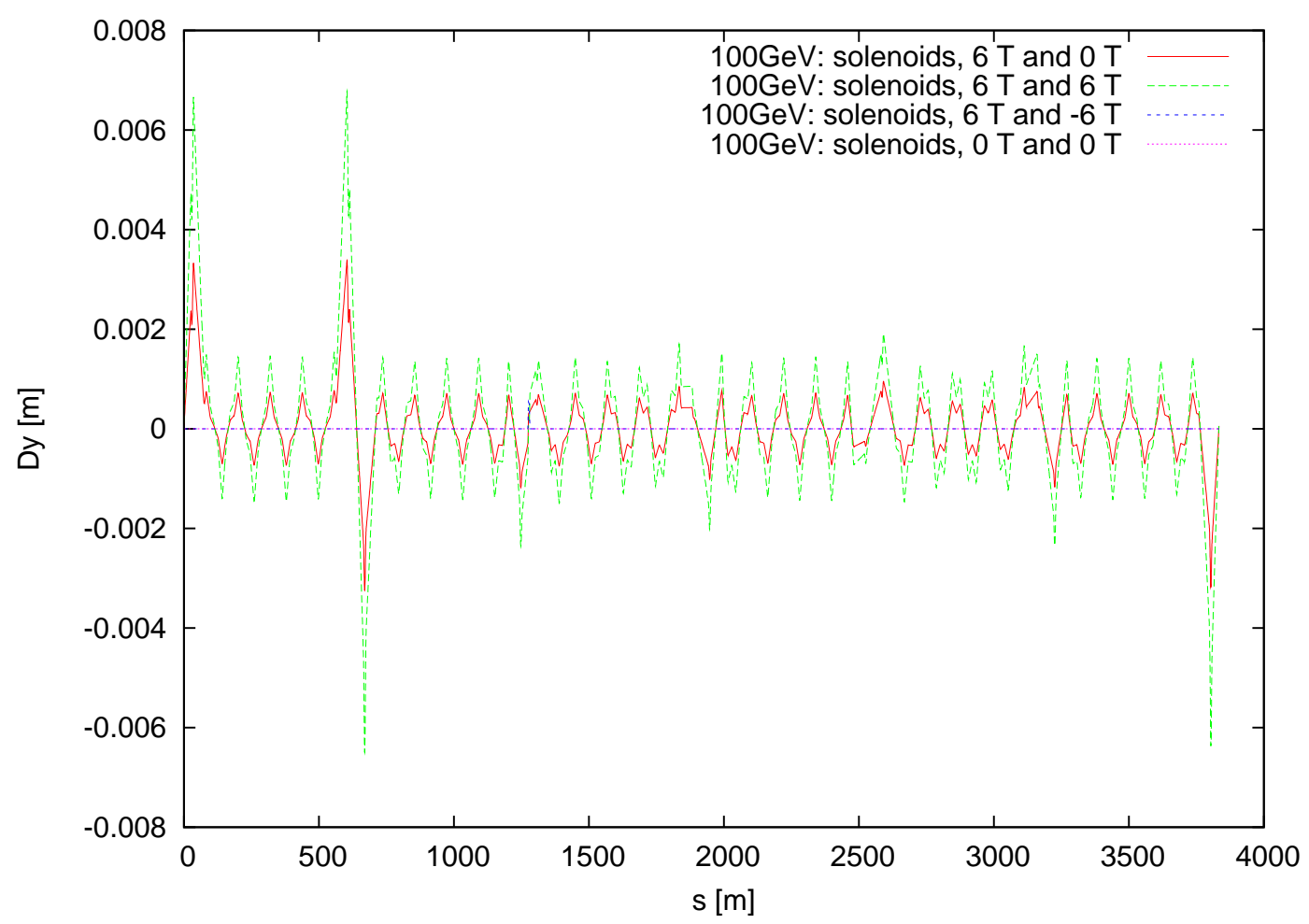

Figure 6: $100 \mathrm{GeV}$ : Vertical dispersion along the ring with different solenoid powering cases 
To numerically calculate the minimum tune split dQmin, we first match the set tunes to $(28.69,29.69)$, then,

$$
d Q \min =\left|C^{-}\right|=\left|Q_{1}-Q_{2}-p\right| .
$$

Following list shows the calculated dQmin for $100 \mathrm{GeV}$ and $250 \mathrm{GeV}$ pp lattices.

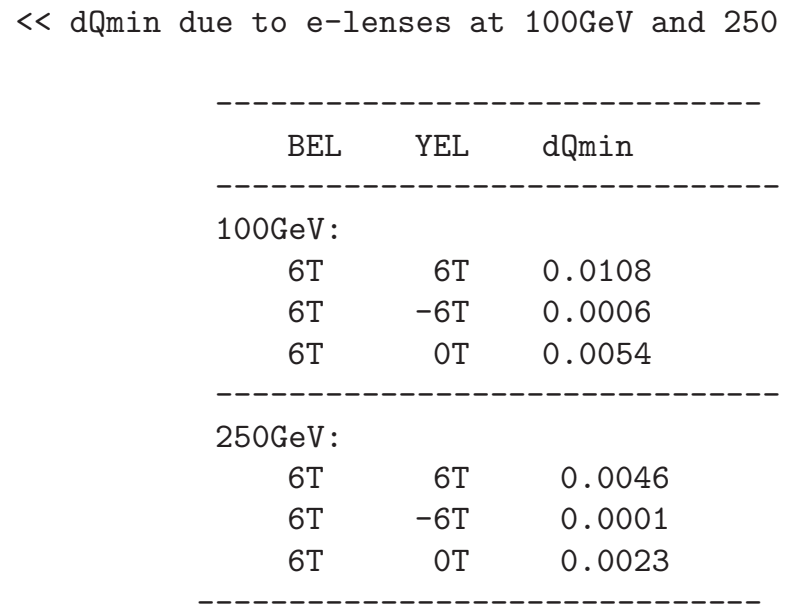

With opposite polarities of the two solenoids, the dQmin are about $1 \times 10^{-4}$ and $6 \times 10^{-4}$ at $100 \mathrm{GeV}$ and $250 \mathrm{GeV}$, which are neglectible for real operation. However, with same polarities of the two solenoids, the dQmin is about 0.005 and 0.01 at $100 \mathrm{GeV}$ and $250 \mathrm{GeV}$, which must be corrected with skew quadrupoles. However, the skew quadrupole almost reach their maximum current limits at $100 \mathrm{GeV}$ and $250 \mathrm{GeV} \mathrm{pp}$ run to correct the residual betatron coupling in the ring. Therefore, in the operation of e-lenses, the two solenoids must be powered with opposite polarities.

\section{Summary}

In this note we calculated the effects of the e-lens solenoids on the proton optics. We found that the effect of these two solenoids on the $\beta$ functions and horizontal dispersion are neglectible. However, regarding to the vertical dispersion and global coupling, we should have the two e-lens solenoids powered with opposite polarities.

\section{References}

[1] Y. Luo and W. Fischer, Outline of using an electron lens for the RHIC head-on beam-beam compensation ( v 0.1 ), BNL C-AD AP Note 286, Dec., 2007.

[2] Y. Luo, Phys. Rev. ST Accel. Beam 7, 124001 (2004).

[3] Y. Luo, NIM A 562 (2006) 57-64.

[4] G. Guignard, Phys. Rev., E 51 (1995) 6104. 


\section{Appendix:}

$<250 \mathrm{GeV}$ : e-lenses' effect at IP6 and IP8 >>

1) BEL and YEL have different polarity

\begin{tabular}{|c|c|c|c|c|c|c|c|c|c|}
\hline \multirow[b]{2}{*}{ BEL } & \multirow[b]{2}{*}{ YEL } & \multirow[b]{2}{*}{ Q1 } & \multirow[b]{2}{*}{ Q2 } & \multicolumn{3}{|c|}{$-------I P 6-----------$} & \multicolumn{3}{|c|}{---------IP8-------- } \\
\hline & & & & beta1 & beta2 & $r$ & beta1 & beta2 & $r$ \\
\hline-6 & 6 & 28.685 & 29.6949 & 0.518719 & 0.51975 & 1 & 0.518796 & 0.519645 & 1 \\
\hline-5 & 5 & 28.6849 & 29.6949 & 0.518745 & 0.519723 & 1 & 0.518799 & 0.519651 & 1 \\
\hline-4 & 4 & 28.6849 & 29.6949 & 0.518767 & 0.519702 & 1 & 0.518801 & 0.519656 & 1 \\
\hline-3 & 3 & 28.6849 & 29.6949 & 0.518784 & 0.519685 & 1 & 0.518803 & 0.519659 & 1 \\
\hline-2 & 2 & 28.6849 & 29.6949 & 0.518796 & 0.519674 & 1 & 0.518805 & 0.519662 & 1 \\
\hline-1 & 1 & 28.6849 & 29.6949 & 0.518803 & 0.519666 & 1 & 0.518805 & 0.519664 & 1 \\
\hline 0 & 0 & 28.6849 & 29.6949 & 0.518806 & 0.519664 & 1 & 0.518806 & 0.519664 & 1 \\
\hline 1 & -1 & 28.6849 & 29.6949 & 0.518803 & 0.519666 & 1 & 0.518805 & 0.519664 & 1 \\
\hline 2 & -2 & 28.6849 & 29.6949 & 0.518796 & 0.519674 & 1 & 0.518805 & 0.519662 & 1 \\
\hline 3 & -3 & 28.6849 & 29.6949 & 0.518784 & 0.519685 & 1 & 0.518803 & 0.519659 & 1 \\
\hline 4 & -4 & 28.6849 & 29.6949 & 0.518767 & 0.519702 & 1 & 0.518801 & 0.519656 & 1 \\
\hline 5 & -5 & 28.6849 & 29.6949 & 0.518745 & 0.519723 & 1 & 0.518799 & 0.519651 & 1 \\
\hline 6 & -6 & 28.685 & 29.6949 & 0.518719 & 0.51975 & 1 & 0.518796 & 0.519645 & 1 \\
\hline
\end{tabular}

2) BEL and YEL have same polarity

\begin{tabular}{|c|c|c|c|c|c|c|c|c|c|}
\hline \multirow[b]{2}{*}{ BEL } & \multirow[b]{2}{*}{ YEL } & \multirow[b]{2}{*}{ Q1 } & \multirow[b]{2}{*}{ Q2 } & \multicolumn{3}{|c|}{----------- IP6------------ } & \multicolumn{3}{|c|}{------------- IP8 -----------} \\
\hline & & & & beta1 & beta2 & $r$ & beta1 & beta2 & $r$ \\
\hline-6 & -6 & 28.6845 & 29.6955 & 0.518748 & 0.519718 & 0.976794 & 0.518825 & 0.519632 & 0.976794 \\
\hline-5 & -5 & 28.6846 & 29.6953 & 0.518766 & 0.519701 & 0.983255 & 0.518819 & 0.519641 & 0.983255 \\
\hline-4 & -4 & 28.6847 & 29.6952 & 0.51878 & 0.519688 & 0.988926 & 0.518815 & 0.51965 & 0.988926 \\
\hline-3 & -3 & 28.6848 & 29.695 & 0.518791 & 0.519677 & 0.993604 & 0.518811 & 0.519656 & 0.993604 \\
\hline-2 & -2 & 28.6849 & 29.695 & 0.518799 & 0.51967 & 0.997101 & 0.518808 & 0.51966 & 0.997101 \\
\hline-1 & -1 & 28.6849 & 29.6949 & 0.518804 & 0.519666 & 0.999267 & 0.518806 & 0.519663 & 0.999267 \\
\hline 0 & 0 & 28.6849 & 29.6949 & 0.518806 & 0.519664 & 1 & 0.518806 & 0.519664 & 1 \\
\hline 1 & 1 & 28.6849 & 29.6949 & 0.518804 & 0.519666 & 0.999267 & 0.518806 & 0.519663 & 0.999267 \\
\hline 2 & 2 & 28.6849 & 29.695 & 0.518799 & 0.51967 & 0.997101 & 0.518808 & 0.51966 & 0.997101 \\
\hline 3 & 3 & 28.6848 & 29.695 & 0.518791 & 0.519677 & 0.993604 & 0.518811 & 0.519656 & 0.993604 \\
\hline 4 & 4 & 28.6847 & 29.6952 & 0.51878 & 0.519688 & 0.988926 & 0.518815 & 0.51965 & 0.988926 \\
\hline 5 & 5 & 28.6846 & 29.6953 & 0.518766 & 0.519701 & 0.983255 & 0.518819 & 0.519641 & 0.983255 \\
\hline 6 & 6 & 28.6845 & 29.6955 & 0.518748 & 0.519718 & 0.976794 & 0.518825 & 0.519632 & 0.976794 \\
\hline
\end{tabular}

3) BEL in on and YEL is off

\begin{tabular}{|c|c|c|c|c|c|c|c|c|c|}
\hline \multirow[b]{2}{*}{ BEL } & \multirow[b]{2}{*}{ YEL } & \multirow[b]{2}{*}{ Q1 } & \multirow[b]{2}{*}{ Q2 } & \multicolumn{3}{|c|}{------------ IP6------------- } & \multicolumn{3}{|c|}{-------------- IP8 ------------} \\
\hline & & & & beta1 & beta2 & $r$ & beta1 & beta2 & $r$ \\
\hline-6 & 0 & 28.6848 & 29.6951 & 0.518798 & 0.519672 & 0.993602 & 0.518771 & 0.519687 & 0.993602 \\
\hline-5 & 0 & 28.6848 & 29.695 & 0.518801 & 0.519669 & 0.99551 & 0.518782 & 0.51968 & 0.99551 \\
\hline-4 & 0 & 28.6849 & 29.695 & 0.518802 & 0.519667 & 0.997101 & 0.51879 & 0.519674 & 0.997101 \\
\hline-3 & 0 & 28.6849 & 29.6949 & 0.518804 & 0.519666 & 0.998358 & 0.518797 & 0.51967 & 0.998358 \\
\hline-2 & 0 & 28.6849 & 29.6949 & 0.518805 & 0.519665 & 0.999267 & 0.518802 & 0.519667 & 0.999267 \\
\hline-1 & 0 & 28.6849 & 29.6949 & 0.518805 & 0.519664 & 0.999816 & 0.518805 & 0.519665 & 0.999816 \\
\hline 0 & 0 & 28.6849 & 29.6949 & 0.518806 & 0.519664 & 1 & 0.518806 & 0.519664 & 1 \\
\hline 1 & 0 & 28.6849 & 29.6949 & 0.518805 & 0.519664 & 0.999816 & 0.518805 & 0.519665 & 0.999816 \\
\hline
\end{tabular}




\begin{tabular}{llllllllll}
2 & 0 & 28.6849 & 29.6949 & 0.518805 & 0.519665 & 0.999267 & 0.518802 & 0.519667 & 0.999267 \\
3 & 0 & 28.6849 & 29.6949 & 0.518804 & 0.519666 & 0.998358 & 0.518797 & 0.51967 & 0.998358 \\
4 & 0 & 28.6849 & 29.695 & 0.518802 & 0.519667 & 0.997101 & 0.51879 & 0.519674 & 0.997101 \\
5 & 0 & 28.6848 & 29.695 & 0.518801 & 0.519669 & 0.99551 & 0.518782 & 0.51968 & 0.99551 \\
6 & 0 & 28.6848 & 29.6951 & 0.518798 & 0.519672 & 0.993602 & 0.518771 & 0.519687 & 0.993602 \\
\hline
\end{tabular}

$<<100 \mathrm{GeV}:$ e-lenses' effect at IP6 and IP8 $>>$

1) BEL and YEL have different polarity

\begin{tabular}{|c|c|c|c|c|c|c|c|c|c|}
\hline \multirow[b]{2}{*}{ BEL } & \multirow[b]{2}{*}{ YEL } & \multirow[b]{2}{*}{ Q1 } & \multirow[b]{2}{*}{ Q2 } & \multicolumn{3}{|c|}{----------IP6--------- } & \multicolumn{3}{|c|}{$-------I P 8----------$} \\
\hline & & & & beta1 & beta2 & $r$ & beta1 & beta2 & $r$ \\
\hline-6 & 6 & 28.6852 & 29.6952 & 0.758689 & 0.77116 & 1 & 0.7594 & 0.769891 & 1 \\
\hline-5 & 5 & 28.6851 & 29.6951 & 0.759034 & 0.770917 & 1 & 0.759528 & 0.770035 & 1 \\
\hline-4 & 4 & 28.685 & 29.695 & 0.759317 & 0.770717 & 1 & 0.759634 & 0.770153 & 1 \\
\hline-3 & 3 & 28.685 & 29.695 & 0.759538 & 0.770562 & 1 & 0.759716 & 0.770245 & 1 \\
\hline-2 & 2 & 28.6849 & 29.6949 & 0.759695 & 0.770451 & 1 & 0.759774 & 0.77031 & 1 \\
\hline-1 & 1 & 28.6849 & 29.6949 & 0.75979 & 0.770385 & 1 & 0.75981 & 0.77035 & 1 \\
\hline 0 & -0 & 28.6849 & 29.6949 & 0.759821 & 0.770363 & 1 & 0.759821 & 0.770363 & 1 \\
\hline 1 & -1 & 28.6849 & 29.6949 & 0.75979 & 0.770385 & 1 & 0.75981 & 0.77035 & 1 \\
\hline 2 & -2 & 28.6849 & 29.6949 & 0.759695 & 0.770451 & 1 & 0.759774 & 0.77031 & 1 \\
\hline 3 & -3 & 28.685 & 29.695 & 0.759538 & 0.770562 & 1 & 0.759716 & 0.770245 & 1 \\
\hline 4 & -4 & 28.685 & 29.695 & 0.759317 & 0.770717 & 1 & 0.759634 & 0.770153 & 1 \\
\hline 5 & -5 & 28.6851 & 29.6951 & 0.759034 & 0.770917 & 1 & 0.759528 & 0.770035 & 1 \\
\hline 6 & -6 & 28.6852 & 29.6952 & 0.758689 & 0.77116 & 1 & 0.7594 & 0.769891 & 1 \\
\hline
\end{tabular}

2) BEL and YEL have same polarity

\begin{tabular}{|c|c|c|c|c|c|c|c|c|c|}
\hline BEL & YEL & Q1 & Q2 & beta1 & $\begin{array}{l}\text { IP6--- } \\
\text { beta2 }\end{array}$ & $r$ & beta1 & beta2 & $r$ \\
\hline-6 & -6 & 28.6828 & 29.6975 & 0.75882 & 0.771272 & 0.916148 & 0.759659 & 0.76971 & 0.916148 \\
\hline-5 & -5 & 28.6834 & 29.6968 & 0.75913 & 0.770998 & 0.933456 & 0.759718 & 0.769902 & 0.933456 \\
\hline-4 & -4 & 28.6839 & 29.6962 & 0.759382 & 0.770772 & 0.951596 & 0.759761 & 0.770064 & 0.951596 \\
\hline-3 & -3 & 28.6843 & 29.6957 & 0.759576 & 0.770594 & 0.969432 & 0.759791 & 0.770192 & 0.969432 \\
\hline-2 & -2 & 28.6846 & 29.6953 & 0.759713 & 0.770466 & 0.985071 & 0.759809 & 0.770286 & 0.985071 \\
\hline-1 & -1 & 28.6848 & 29.695 & 0.759794 & 0.770389 & 0.996027 & 0.759818 & 0.770343 & 0.996027 \\
\hline 0 & 0 & 28.6849 & 29.6949 & 0.759821 & 0.770363 & 1 & 0.759821 & 0.770363 & 1 \\
\hline 1 & 1 & 28.6848 & 29.695 & 0.759794 & 0.770389 & 0.996027 & 0.759818 & 0.770343 & 0.996027 \\
\hline 2 & 2 & 28.6846 & 29.6953 & 0.759713 & 0.770466 & 0.985071 & 0.759809 & 0.770286 & 0.985071 \\
\hline 3 & 3 & 28.6843 & 29.6957 & 0.759576 & 0.770594 & 0.969432 & 0.759791 & 0.770192 & 0.969432 \\
\hline 4 & 4 & 28.6839 & 29.6962 & 0.759382 & 0.770772 & 0.951596 & 0.759761 & 0.770064 & 0.951596 \\
\hline 5 & 5 & 28.6834 & 29.6968 & 0.75913 & 0.770998 & 0.933456 & 0.759718 & 0.769902 & 0.933456 \\
\hline 6 & 6 & 28.6828 & 29.6975 & 0.75882 & 0.771272 & 0.916148 & 0.759659 & 0.76971 & 0.916148 \\
\hline
\end{tabular}

3) BEL in on and YEL is off

$\begin{array}{ccccccccccc}\text { BEL YEL } & \text { Q1 } & \text { Q2 } & \text { beta1 } & \text { beta2 } & \text { r } & \text { beta1 } & \text { beta2 } & \text { r } \\ -6 & 6 & 28.6852 & 29.6952 & 0.758689 & 0.77116 & 1 & 0.7594 & 0.769891 & 1 \\ -5 & 5 & 28.6851 & 29.6951 & 0.759034 & 0.770917 & 1 & 0.759528 & 0.770035 & 1 & \end{array}$




\begin{tabular}{|c|c|c|c|c|c|c|c|c|c|}
\hline-4 & 4 & 28.685 & 29.695 & 0.759317 & 0.770717 & 1 & 0.759634 & 0.770153 & 1 \\
\hline-3 & 3 & 28.685 & 29.695 & 0.759538 & 0.770562 & 1 & 0.759716 & 0.770245 & 1 \\
\hline-2 & 2 & 28.6849 & 29.6949 & 0.759695 & 0.770451 & 1 & 0.759774 & 0.77031 & 1 \\
\hline-1 & 1 & 28.6849 & 29.6949 & 0.75979 & 0.770385 & 1 & 0.75981 & 0.77035 & 1 \\
\hline 0 & -0 & 28.6849 & 29.6949 & 0.759821 & 0.770363 & 1 & 0.759821 & 0.770363 & 1 \\
\hline 1 & -1 & 28.6849 & 29.6949 & 0.75979 & 0.770385 & 1 & 0.75981 & 0.77035 & 1 \\
\hline 2 & -2 & 28.6849 & 29.6949 & 0.759695 & 0.770451 & 1 & 0.759774 & 0.77031 & 1 \\
\hline 3 & -3 & 28.685 & 29.695 & 0.759538 & 0.770562 & 1 & 0.759716 & 0.770245 & 1 \\
\hline 4 & -4 & 28.685 & 29.695 & 0.759317 & 0.770717 & 1 & 0.759634 & 0.770153 & 1 \\
\hline 5 & -5 & 28.6851 & 29.6951 & 0.759034 & 0.770917 & 1 & 0.759528 & 0.770035 & 1 \\
\hline 6 & -6 & 28.6852 & 29.6952 & 0.758689 & 0.77116 & 1 & 0.7594 & 0.769891 & 1 \\
\hline
\end{tabular}

